\title{
Validation of standard neutron data libraries for LWR storage pools and transport casks criticality safety evaluations
}

\author{
Edwin Kolbe ${ }^{\text {a }}$, Alexander Vasiliev, and Martin A. Zimmermann \\ Laboratory for Reactor Physics and Systems Behaviour, Paul Scherrer Institut, 5232-Villigen PSI, Switzerland
}

\begin{abstract}
This paper addresses the assessment of MCNPX-2.5.0 criticality calculations using continuous energy cross section libraries based on JEF-2.2, JENDL-3.3, ENDF/B-6.8, and the recently released JEFF-3.1 data files. For this purpose, validation calculations were performed for a suite of benchmarks from the International Handbook of Evaluated Criticality Safety Benchmark Experiments. The benchmarks were selected on the basis of their similarity to designs found in today's Light Water Reactor (LWR) compact storage pools and transport casks, including MOX fuel rod assemblies, and in total comprised as much as 149 cases. In order to define the ranges of applicability of the used calculational methods and to detect possible trends, the spectrum-related characteristics of the modeled critical experimental configurations were analysed. It is found that the recently released JEFF-3.1 library improves the older JEF-2.2 compilation and leads to a value for the weighted average of the normalized eigenvalues $\left\langle\mathrm{k}_{\mathrm{eff}}^{\text {calc }} / \mathrm{k}_{\mathrm{eff}}^{\text {exp }}\right\rangle$ very close to unity. All libraries have in common that any evidence of a statistically significant trend in the normalized eigenvalues versus both spectrum-related characteristics and experimental design parameters could not be found.
\end{abstract}

\section{Introduction}

A methodology for criticality safety evaluations (CSE) using the Monte Carlo radiation transport code MCNPX is currently under development within the STARS project at LRS/PSI. Following nuclear criticality safety practices the validity of a calculational method (consisting of the computer code and the cross section data) must be established by correlating the experimental results of a suitable set of critical experiments with the calculational results obtained for these same systems [1]. In this context (and as a first step) an analysis of the MCNPX-2.4.0/JEF-2.2 and JENDL-3.3 results for a suite of 15 low-enriched thermal compound uranium benchmarks was already performed at PSI [2], which, in total, comprised the evaluation of 105 cases representing wet storage and transport casks. Recently (as a second step) the first analysis has been enhanced by also applying the ENDF/B-6.8 neutron data library, studying spectrum related trends, and, furthermore, employing the most recent official release of the code (MCNPX-2.5.0 [3]) with an improved $\mathrm{S}(\alpha, \beta)$ thermal neutron scattering treatment [4]. In the work presented here the former analyses are extended by:

- including a cross section library based on the recently released JEFF-3.1 [5] data files,

- adding 17 more cases in order to include the structural materials that could be employed in dry and wet storage pools,

- adding 27 MOX cases.

The data obtained form a basis for the establishment of the PSI CSE methodology.

\footnotetext{
${ }^{a}$ Presenting author, e-mail: Edwin.Kolbe@psi.ch
}

\section{Methodology}

All results presented in this paper have been calculated with the latest official version of MCNPX, which is MCNPX2.5.0 [3], released in April 2005. The four continuous energy neutron data libraries (ENDF/B-6.8 [6], JEF-2.2 [7], JEFF3.1 [5], and JENDL-3.3 [8]) that were applied are distributed by the NEA/OECD Data Bank Computer Program Service. In the perspective of assessing possible changes by new releases of a specific library, we also applied the somewhat older JEF2.2 library.

All critical experiments were extracted from the "International Handbook of Evaluated Criticality Safety Benchmark Experiments" [9]. The selection of the benchmark configurations was based on their similarity to designs found in today's LWR compact dry storage pools and transport casks. The original suite of 15 low-enriched thermal compound (LCT) uranium benchmarks obtained in ref. [2] comprised the evaluation of 105 cases representing wet storage and transport casks. This set was extended by 44 additional cases in order to include both the structural materials (boral, boroflex) that could be employed in dry storage pools (17 cases) and MOXfuel (27 cases). The list of the added benchmark configurations with indication of some experimental design characteristics is presented in table 1 .

In the first studies [2] a deviation of the distribution of the normalized eigenvalues (i.e., the ratio $\mathrm{k}_{\mathrm{eff}}^{\mathrm{calc}} / \mathrm{k}_{\mathrm{eff}}^{\mathrm{exp}}$ of the calculated to experimental results) from a Gaussian distribution was observed, particularly, if very accurate Monte Carlo criticality calculations had been performed. It was conjectured that the non-normality could be caused by nonspecified experiment/evaluation-related biases in the $\mathrm{k}_{\mathrm{eff}}^{\exp }$ values. Thus the question arose whether an analysis based on the assumption of normality of the $\mathrm{k}_{\mathrm{eff}}^{\text {calc }} / \mathrm{k}_{\mathrm{eff}}^{\text {exp }}$ distribution (which is frequently used for practical CSE due to its easiness and flexibility of application) is really reliable. Because of such concerns, we will apply in the present study also the 
Table 1. Some design characteristics for the additional evaluated benchmark experiments (LCT $=$ Low-enriched-Compound-Thermal benchmark, MCT = Mixed-Compound-Thermal benchmark [9]).

\begin{tabular}{lllll}
\hline ID & cases & $\begin{array}{l}\text { fuel, } \\
\text { enrichment }\end{array}$ & $\begin{array}{l}\text { pitch } \\
{[\mathrm{cm}]}\end{array}$ & $\begin{array}{l}\text { Laboratory, year } \\
\text { of experiment }\end{array}$ \\
\hline LCT-13 & $3+4$ & ${ }^{235} \mathrm{U}, 4.31 \%$ & 1.892 & PNL, USA, 1979 \\
LCT-16 & $12 \div 14$ & ${ }^{235} \mathrm{U}, 2.35 \%$ & 2.032 & PNL, USA, 1977 \\
LCT-42 & $3+4$ & ${ }^{235} \mathrm{U}, 2.35 \%$ & 1.684 & PNL, USA, 1980 \\
LCT-51 & $10 \div 19$ & ${ }^{235} \mathrm{U}, 2.46 \%$ & 1.636 & B\&W, USA, 1978 \\
\hline MCT-1 & $1 \div 4$ & $\mathrm{Pu}, 19.7 \%$ & $0.952 \div 1.91$ & PNL, USA, 1978 \\
MCT-2 & $1 \div 6$ & $\mathrm{Pu}, 2.0 \%$ & $1.779 \div 2.51$ & PNL, USA, 1976 \\
MCT-3 & $1 \div 6$ & $\mathrm{Pu}, 6.6 \%$ & $1.321 \div 2.64$ & WREC, USA, 1965 \\
MCT-4 & $1 \div 11$ & $\mathrm{Pu}, 3.0 \%$ & $1.825 \div 2.47$ & JAERI, Japan, 1975 \\
\hline
\end{tabular}

distribution-free estimates for $\mathrm{k}_{\mathrm{eff}}$ lower tolerance bounds (LTB) and will compare them with the LTBs based on the normality of the calculated results. Fortunately, the differences between these two approaches will turn out to be insignificant from a practical point of view.

\section{Results and analysis}

\subsection{Statistical description}

In the (statistical) evaluations the normalized eigenvalues $\mathrm{k}_{\mathrm{c}, \mathrm{i}}=\mathrm{k}_{\mathrm{eff}, \mathrm{i}}^{\text {calc }} / \mathrm{k}_{\mathrm{eff}, \mathrm{i}}^{\text {exp }}$ will be used, and the following formulas from statistics were applied to analyse the $\mathrm{k}_{\mathrm{eff}}^{\text {calc }} / \mathrm{k}_{\mathrm{eff}}^{\mathrm{exp}}$ sample:

$$
\begin{gathered}
\left\langle k_{c}\right\rangle=\frac{1}{w} \sum_{i=1}^{N} w_{i}\left(k_{c, i}\right), \\
\sigma^{\prime}=\frac{1}{\sqrt{w}} ; \quad w=\sum_{i=1}^{N} w_{i} ; \quad w_{i}=\frac{1}{\sigma_{i}^{2}}, \\
\sigma_{i}=\left(k_{c, i}\right) \sqrt{\left(\frac{\sigma_{i}^{\exp }}{k_{\mathrm{eff}, \mathrm{i}}^{\exp }}\right)^{2}+\left(\frac{\sigma_{i}^{M C}}{k_{\mathrm{eff}, \mathrm{i}}^{\mathrm{calc}}}\right)^{2}}, \\
s=\sqrt{\frac{1}{(N-3)} \sum_{i=1}^{N}\left(\left(k_{c, i}\right)-\left\langle k_{c}\right\rangle\right)^{2} .}
\end{gathered}
$$

Equations (1) and (2) give, respectively, the weighted average $\left\langle\mathrm{k}_{\mathrm{c}}\right\rangle$-value and its standard deviation $\sigma^{\prime}$. The weights $w_{i}$ are proportional to $1 / \sigma_{i}^{2}$, where $\sigma_{i}$ (eq. (3)) is the uncertainty of a single observation and incorporates the uncertainty from both the benchmark $\left(\sigma_{i}^{\exp }\right)$ and the calculation $\left(\sigma_{i}^{M C}\right)$. Finally $s$ (eq. (4)) denotes the sample standard deviation and will be used later on to evaluate the LTB based on the assumption of normality of the $\mathrm{k}_{\mathrm{eff}}^{\text {calc }} / \mathrm{k}_{\mathrm{eff}}^{\text {exp }}$ population. Therefore we applied equation (4), which gives the best estimate of the standard deviation when a Gaussian distribution is assumed (see ICSBEP Guide to the Expression of Uncertainties from ref. [9]).

The statistical analysis was first separately applied to the two samples consisting of the 17 additional LCT-cases and the 27 MCT-cases listed in table 1 . The values obtained for
Table 2. Statistical description of the results obtained by evaluating only the 17 additional LCT cases with four cross section libraries.

\begin{tabular}{lllll}
\hline $\begin{array}{l}\text { Cross section } \\
\text { Library }\end{array}$ & $\left\langle k_{c}\right\rangle \pm \sigma^{\prime}$ & $\begin{array}{l}\text { Min }_{\mathrm{c}, \mathrm{i}} \\
\pm \sigma_{i}\end{array}$ & $\begin{array}{l}\operatorname{Max~k}_{\mathrm{c}, \mathrm{i}} \\
\pm \sigma_{i}\end{array}$ & $\mathrm{~s}$ \\
\hline ENDF/B-6.8 & 0.9903 & 0.9844 & 0.9940 & 0.0035 \\
& \pm 0.0005 & \pm 0.0019 & \pm 0.0018 & \\
$\mathrm{JEF}-2.2$ & 0.9943 & 0.9875 & 0.9994 & 0.0042 \\
& \pm 0.0005 & \pm 0.0019 & \pm 0.0018 & \\
JEFF-3.1 & 0.9958 & 0.9894 & 1.0012 & 0.0042 \\
& \pm 0.0005 & \pm 0.0019 & \pm 0.0018 & \\
JENDL-3.3 & 0.9937 & 0.9874 & 0.9990 & 0.0039 \\
& \pm 0.0005 & \pm 0.0019 & \pm 0.0018 & \\
\hline
\end{tabular}

Table 3. Statistical description of the results obtained by evaluating only the 27 additional MCT cases with four cross section libraries.

\begin{tabular}{lllll}
\hline $\begin{array}{l}\text { Cross section } \\
\text { Library }\end{array}$ & $\left\langle k_{c}\right\rangle \pm \sigma^{\prime}$ & $\begin{array}{l}\operatorname{Min}_{\mathrm{c}, \mathrm{i}} \\
\pm \sigma_{i}\end{array}$ & $\begin{array}{l}\operatorname{Max~k}_{\mathrm{c}, \mathrm{i}} \\
\pm \sigma_{i}\end{array}$ & $\mathrm{~s}$ \\
\hline ENDF/B-6.8 & 0.9948 & 0.9899 & 0.9992 & 0.0026 \\
& \pm 0.0006 & \pm 0.0046 & \pm 0.0021 & \\
$\mathrm{JEF}-2.2$ & 0.9974 & 0.9939 & 0.9997 & 0.0020 \\
& \pm 0.0006 & \pm 0.0059 & \pm 0.0028 & \\
JEFF-3.1 & 0.9995 & 0.9960 & 1.0035 & 0.0022 \\
& \pm 0.0006 & \pm 0.0046 & \pm 0.0021 & \\
JENDL-3.3 & 0.9993 & 0.9944 & 1.0030 & 0.0025 \\
& \pm 0.0006 & \pm 0.0046 & \pm 0.0021 & \\
\hline
\end{tabular}

all libraries are presented in table 2 and table 3, respectively, and should both be compared with the corresponding results calculated for the set of 105 benchmarks used in the previous study [4] and shown in table 4.

The comparisons show that although the weighted averages obtained for the additional 27 MCT-cases are slightly greater, they yet are in good agreement with the weighted averages obtained for the original suite of benchmarks. But, for all libraries, the weighted averages obtained for the additional 17 LCT-cases are notably smaller than the weighted averages calculated for the set of 105 benchmarks before. At first glance one may take this difference as an indication that something is wrong with cross sections related to boron, which is contained in the separation material of the additional cases. However, this would have to apply to all libraries in equal measure, which is unlikely. Studying the calculated eigenvalues separately for all independently evaluated experiments shows a general trend: the $\mathrm{k}_{\mathrm{eff}}^{\mathrm{cal}}$ values for many of the cases belonging to LCT-11 and LCT-51 (both performed at the same facility) lie relatively low in comparison to all other calculated eigenvalues. The original set of 105 benchmarks contained only 12 cases from LCT-11 and LCT-51 [2]. Contrary, 10 of the additional 17 LCT cases belong to LCT-51, which is a significantly higher fraction. The difference in the weighted average of the 17-LCT and the original 105 benchmarks is simply explained by this fact. Thus, as significant differences between the three $\mathrm{k}_{\mathrm{eff}}^{\mathrm{calc}} / \mathrm{k}_{\mathrm{eff}}^{\mathrm{exp}}$ samples could not be found, a statistical analysis was performed for the combined set (comprising 149 cases). Results are listed in table 5. 
Table 4. Statistical description of the results obtained by evaluation of the original set of 105 benchmark experiments with four cross section libraries.

\begin{tabular}{lllll}
\hline $\begin{array}{l}\text { Cross section } \\
\text { Library }\end{array}$ & $\left\langle k_{c}\right\rangle \pm \sigma^{\prime}$ & $\begin{array}{l}\operatorname{Min}_{\mathrm{c}, \mathrm{i}} \\
\pm \sigma_{i}\end{array}$ & $\begin{array}{l}\operatorname{Max~}_{\mathrm{c}, \mathrm{i}} \\
\pm \sigma_{i}\end{array}$ & $\mathrm{~s}$ \\
\hline ENDF/B-6.8 & 0.9928 & 0.9874 & 0.9998 & 0.0028 \\
& \pm 0.0002 & \pm 0.0018 & \pm 0.0020 & \\
$\mathrm{JEF}-2.2$ & 0.9964 & 0.9881 & 1.0026 & 0.0031 \\
& \pm 0.0002 & \pm 0.0018 & \pm 0.0020 & \\
$\mathrm{JEFF}-3.1$ & 0.9993 & 0.9921 & 1.0049 & 0.0031 \\
& \pm 0.0002 & \pm 0.0018 & \pm 0.0016 & \\
$\mathrm{JENDL}-3.3$ & 0.9966 & 0.9903 & 1.0022 & 0.0028 \\
& \pm 0.0002 & \pm 0.0018 & \pm 0.0020 & \\
\hline
\end{tabular}

Table 5. Statistical description of the results obtained by evaluation of the combined set of 149 benchmark experiments with four cross section libraries.

\begin{tabular}{lllll}
\hline $\begin{array}{l}\text { Cross section } \\
\text { Library }\end{array}$ & $\left\langle k_{c}\right\rangle \pm \sigma^{\prime}$ & $\begin{array}{l}\operatorname{Min}_{\mathrm{c}, \mathrm{i}} \\
\pm \sigma_{i}\end{array}$ & $\begin{array}{l}\operatorname{Max~}_{\mathrm{c}, \mathrm{i}} \\
\pm \sigma_{i}\end{array}$ & $\begin{array}{l}\mathrm{s} / \text { bias } \\
{[\mathrm{pcm}]}\end{array}$ \\
\hline ENDF/B-6.8 & 0.9927 & 0.9844 & 0.9998 & 0.0029 \\
& \pm 0.0002 & \pm 0.0019 & \pm 0.0020 & -730 \\
$\mathrm{JEF}-2.2$ & 0.9962 & 0.9875 & 1.0026 & 0.0031 \\
& \pm 0.0002 & \pm 0.0019 & \pm 0.0020 & -380 \\
$\mathrm{JEFF}-3.1$ & 0.9990 & 0.9894 & 1.0049 & 0.0032 \\
& \pm 0.0002 & \pm 0.0019 & \pm 0.0016 & -100 \\
$\mathrm{JENDL}-3.3$ & 0.9965 & 0.9874 & 1.0022 & 0.0031 \\
& \pm 0.0002 & \pm 0.0019 & \pm 0.0020 & -350 \\
\hline
\end{tabular}

\subsection{Comparison of Libraries}

The bias, i.e., the systematic differences between calculational method results and experimental data is given by $\mathrm{b}=1.0-\left\langle\mathrm{k}_{\mathrm{c}}\right\rangle$ and was calculated for each of the cross section libraries. The results are listed in the last column of table 5. Except for the ENDF/B-6.8 data set, the agreement of the calculational methods with the benchmark cases is quite satisfactory. Especially the JEFF-3.1 library leads to a very small bias. The results obtained with the JEF-2.2 and JENDL-3.3 libraries are in good agreement and only slightly smaller than 1.0. The standard deviations of all libraries have values close to $300 \mathrm{pcm}$. It is interesting to study the effect that relatively recent updates of a library can have on the normalized eigenvalues by comparing the results for JEF-2.2 and its successor JEFF-3.1. The values in table 5 show that the bias may change by as much as $\sim 300 \mathrm{pcm}$ (but the standard deviation stays roughly constant).

\subsection{Analysis of trends}

The relationship between the normalized eigenvalues $\mathrm{k}_{\mathrm{eff}}^{\mathrm{calc}} / \mathrm{k}_{\mathrm{eff}}^{\mathrm{exp}}$ and the following experimental benchmark design parameters were investigated: fuel enrichment, moderation ratio, fuel rod pitch size and fuel pellet diameter. Statistically significant trends could not be found for all parameters studied.

The spectrum-related trends we studied included (as recommended in ref. [9]) the observables: the energy corresponding to the average neutron lethargy causing fission (EALF),
Table 6. LTBs to contain at least the proportion $p$ of a normally distributed $\mathrm{k}_{\mathrm{eff}}^{\text {calc }} / \mathrm{k}_{\mathrm{eff}}^{\text {exp }}$ population with a confidence level $\alpha$.

\begin{tabular}{lcccc}
\hline Proportion $p$ & $90 \%$ & $95 \%$ & $95 \%$ & $99 \%$ \\
Confidence $\alpha$ & $90 \%$ & $95 \%$ & $99 \%$ & $99 \%$ \\
\hline Coverage factor k1 & 1.43 & 1.87 & 1.97 & 2.74 \\
\hline ENDF/B-6.8 & 0.9885 & 0.9872 & 0.9869 & 0.9847 \\
JEF-2.2 & 0.9919 & 0.9905 & 0.9902 & 0.9878 \\
JEFF-3.1 & 0.9944 & 0.9929 & 0.9926 & 0.9901 \\
JENDL-3.3 & 0.9920 & 0.9907 & 0.9903 & 0.9880 \\
\hline
\end{tabular}

the average neutron energy causing fission (AFGE), the neutron gas temperature in the thermal energy range $\left(\mathrm{T}_{n}\right)$, the percentage of the neutron flux, fissions, and captures that occur in a three- (thermal, intermediate, fast) and thirty-group energy mesh, the percentage of fissions (of ${ }^{235} \mathrm{U},{ }^{238} \mathrm{U}$ ) and captures by isotopes $\left({ }^{235} \mathrm{U},{ }^{238} \mathrm{U},{ }^{1} \mathrm{H},{ }^{16} \mathrm{O},{ }^{27} \mathrm{Al}\right)$ over the core region, and the number of average fission neutrons produced per neutron absorbed in the core $\left(v \Sigma_{f} / \Sigma_{a}\right)$. For all four cross section libraries, no significant trends could be identified.

The ranges of the observables, as calculated with the different libraries, are generally found to be in good agreement, except for captures on ${ }^{16} \mathrm{O}$. Here the range calculated with the JENDL-3.3 library does not overlap with the ranges obtained with ENDF/B-6.8, JEF-2.1 and JEFF-3.1. However, this does not have a significant effect on the calculated $\mathrm{k}_{\mathrm{eff}}$-eigenvalues. For other applications, the situation can be quite different: the consequences of notably less captures on ${ }^{16} \mathrm{O}$ using JENDL3.3 resulting in higher fast neutron fluence is discussed in ref. [10].

\subsection{Estimation of a lower tolerance bound for $\mathbf{k}_{\mathrm{eff}}$}

According to ref. [11] the (one-sided) LTB to be exceeded by at least a proportion $\mathrm{p}$ of a normal $\mathrm{k}_{\mathrm{eff}}^{\mathrm{calc}} / \mathrm{k}_{\mathrm{eff}}^{\mathrm{exp}}$ population is:

$$
K_{e f f}^{L T B}=\left\langle k_{c}\right\rangle-k_{l}(\alpha, p, N) \cdot s,
$$

where the coverage factor $k_{1}$ depends on the confidence level $(1-\alpha)$, the proportion of the population $p$, and the sample size $N$. It can be found in statistical data tables [11]. The LTBs calculated following equation (5) are listed in table 6 for different values of proportion $p$ and confidence $\alpha$.

To determine the LTB for the $\mathrm{k}_{\mathrm{eff}}^{\mathrm{calc}} / \mathrm{k}_{\mathrm{eff}}^{\exp }$ sample based on a distribution-free approach, order statistics must be applied [11]. Accordingly the LTB is given by the endpoint $\left(\mathrm{k}_{\mathrm{eff}, L}^{\text {calc }} / \mathrm{k}_{\mathrm{eff}, L}^{\mathrm{exp}}\right)$ of the ordered sample with size $N$, and $L$ chosen as the largest integer such that:

$$
1-\sum_{I=0}^{l-1} \frac{N !}{i !(N-i) !} p^{N-i}(1-p)^{i} \geq \alpha .
$$

The correspondingly calculated LTBs are given in table 7, again for different values of the proportion $p$ and the confidence $\alpha$. 
Table 7. LTBs to contain at least the proportion $p$ of a $\mathrm{k}_{\mathrm{eff}}^{\text {calc }} / \mathrm{k}_{\mathrm{eff}}^{\exp }$ population with a confidence $\alpha$ based on order statistics.

\begin{tabular}{cccc}
\hline Proportion $p$ & $90 \%$ & $95 \%$ & $95 \%$ \\
Confidence $\alpha$ & $93.6 \%$ & $94.3 \%$ & $99.5 \%$ \\
\hline L & 10 & 4 & 2 \\
\hline ENDF/B-6.8 & 0.9880 & 0.9872 & 0.9850 \\
JEF-2.2 & 0.9915 & 0.9888 & 0.9881 \\
JEFF-3.1 & 0.9936 & 0.9917 & 0.9896 \\
JENDL-3.3 & 0.9913 & 0.9900 & 0.9877 \\
\hline
\end{tabular}

\section{Discussion and conclusion}

Four continuous-energy neutron data libraries have been assessed for LWR criticality safety applications using the Monte Carlo radiation transport code MCNPX-2.5.0. For a suite of 149 low-enriched thermal compound uranium benchmarks the calculated $\mathrm{k}_{\mathrm{eff}}$-values were generally found to be in good agreement (within $\pm 2 \sigma$ ) with the reference benchmark values. The weighted average $\left\langle\mathrm{k}_{\mathrm{c}}\right\rangle$ of all benchmarks applying the ENDF/B-6.8, JEF-2.1, JEFF-3.1 and JENDL3.3 libraries slightly underestimates criticality by $730 \mathrm{pcm}$, $380 \mathrm{pcm}, 100 \mathrm{pcm}$ and $350 \mathrm{pcm}$, respectively, which confirms the long-standing problem of low eigenvalues in waterreflected low-enriched-uranium fuel lattice systems [12], before the JEFF-3.1 and ENDF/B-7.0 releases [13]. Also an analysis of trends related to experimental design parameters and of spectrum-related trends has been performed. As statistically significant trends could not be found, there is no need to include trends in future determinations of an upper subcriticality limit (USL) using MCNPX-2.5.0 and the assessed libraries. Due to the absence of trends, moderate extrapolations outside the range of validation of the sets of Monte Carlo Code/cross section libraries may be well justifiable. The absence of statistical significant trends further supports the statement that the described calculational method appears to be a reliable tool to analyse critical configurations relevant to LWR CSE.
The comparison of the results obtained with four cross section libraries showed that the weighted average $\left\langle\mathrm{k}_{\mathrm{c}}\right\rangle$ calculated with the recently released JEFF-3.1 [5] data files was in closest agreement with the benchmarks. Finally we also confirmed the findings from previous analyses $[2,4]$ that the differences between the LTBs derived based on a distributionfree approach and those estimated based on the assumption of normality of the $\mathrm{k}_{\mathrm{eff}}^{\mathrm{calc}} / \mathrm{k}_{\mathrm{eff}}^{\mathrm{exp}}$ population turn out to be small for all 4 libraries used. Thus, the usual practice of basing CSE on the assumption of normality of the $\mathrm{k}_{\mathrm{eff}}^{\text {calc }} / \mathrm{k}_{\mathrm{eff}}^{\exp }$ distribution appears to be acceptable.

This work has been partly supported by swissnuclear, the organization of the Swiss nuclear utilities.

\section{References}

1. ANSI/ANS-8.1-1998 (ANS, La Grange Park, Illinois, 1998).

2. A.V. Vasiliev, J.R. Lebenhaft, M.A. Zimmermann, in Proceedings of the "MEGC-2005" International Topical Meeting (Avignon, France, 2005), on CD-ROM, paper 165.

3. D.B. Pelowitz (ed.), MCNPX User's Manual, Version 2.5.0., LACP-05-0369 (LANL, 2005).

4. E. Kolbe, A. Vasiliev, M.A. Zimmermann, in Proceedings of the ANS Topical Meeting on Reactor Physics (Physor-2006), Vancouver, BC, Canada, 2006, on CD-ROM, paper D081.

5. ZZ-MCJEFF3.1NEA, NEA-1768/01-11, OECD/NEA Data Bank (Paris, France, 2006).

6. ZZ-MCB-ENDF/B-6.8, NEA-1669/03, ibid. (2004).

7. ZZ-MCJEF22NEA.BOLIB, NEA-1616/04, ibid. (2001).

8. ZZ-FSXLIBJ33, NEA-1424/04, ibid. (2003).

9. ICSBEP-2005, NEA-1486/04, ibid. (2005).

10. A. Vasiliev et al. (these proceedings).

11. G.J. Hahn, W.Q. Meeker, Statistical Intervals. A Guide for Practitioners (John Wiley \& Sons, 1991).

12. A.C. Kahler, Nucl. Sci. Eng. 145, 213 (2003).

13. S.C. van der Marck, Nucl. Data Sheets 107, 3061 (2006). 